\title{
Vers une diminution de la vulnérabilité du bâti face au risque industriel majeur - L'exemple des prescriptions de travaux issues du Plan de Prévention des Risques Technologiques (PPRT) français
}

\author{
Towards a reduction of the vulnerability of the building against the major \\ industrial risk - The example of the work prescriptions resulting from the
} French Technological Risk Prevention Plan (PPRT)

\author{
Jean-Christophe Blesius ${ }^{1}$ \\ ${ }^{1}$ Chercheur associé au Lab'Urba de l'université de Paris-Est, France, jc.blesius@gmail.com
}

RÉSUMÉ. En France, les industries à risque cohabitent parfois de manière étroite avec des résidences. Cet article propose de mener une réflexion sur la construction, complexe, de la législation en matière de maîtrise de l'urbanisation. Cette dernière est le résultat, entre autres, d'une volonté des pouvoirs publics de garantir un développement industriel efficace ; ainsi que du retour d'expérience des catastrophes industrielles qui se sont produites au cours de l'histoire. Le propos porte ensuite la focale sur l'instrument récent qu'est le Plan de Prévention des Risques Technologiques (PPRT). Ce dernier impose notamment aux riverains d'installations à risque d'effectuer des travaux de sécurisation dans leurs logements. Bien que leur prise en charge financière ait fait l'objet de nombreux débats, cette disposition témoigne de l'aboutissement d'un processus qui conduit à envisager ces territoires comme un tout. Les premiers résultats de cette recherche tendent à montrer qu'aujourd'hui, les différents maillons ne se tournent plus le dos et se greffent ensemble pour former une chaîne au service du « vivre avec » le risque industriel.

ABSTRACT. In France, high-risk industries sometimes have to coexist with nearby residential areas. This article aims to reflect on the complex process of the legislation on the control of urbanization, which is the result of a willingness of the authorities to ensure effective industrial development while learning from past experiences of industrial disasters. The article will focus on Technological Risk Prevention Plan (TRPP), a recently developed tool which populations living near high-risk facilities can use to carry out assessments to protect their homes from risk. Although financial support has been the subject of much debate, this situation reflects the outcome of a process that leads to consider these territories as a whole. The first results of this research suggest that today industry and residents can work together to make the most of a situation which involves living in risk.

MOTS-CLÉS. maîtrise de l'urbanisation, plan de prévention des risques technologiques, risques industriels.

KEYWORDS. urbanization control, technological risk prevention plan, industrial risks.

\section{Introduction}

Depuis plusieurs années, certaines industries se sont associées pour travailler et réfléchir ensemble à la manière dont elles peuvent interagir pour diminuer leur empreinte sur l'environnement. Certains auteurs y voient l'expression d'un écosystème industriel vecteur de développement durable, voire d'une " écologie industrielle » (DIEMER, LABRUNE, 2007). Cette volonté, pour ne donner qu'un exemple, est présente au sein de l'importante zone industrielle de Montréal-Est (Québec) (BLESIUS, 2014, pp. 340-341). Est-il néanmoins possible d'élargir la réflexion en englobant les territoires contigus aux industries (NOVEMBER, 2002) ? Des territoires supports d'habitations notamment. En effet, l'existence de risques industriels majeurs - à savoir la probabilité que survienne une explosion, une fuite toxique ou un incendie 
amène les acteurs de la ville à réfléchir aux réponses qui permettraient d'améliorer les conditions de vie des individus vivant autour de ces entités. Le présent article propose d'explorer la problématique suivante :

L'analyse des risques majeurs ${ }^{1}$, de leurs effets; les réflexions sur les manières de réduire la vulnérabilité peuvent-elles avoir une conséquence sur la qualité des logements, voire sur leur résilience ?

Pour y répondre, deux contextes particuliers seront pris en considération : celui du contact entre des industries à risque et des constructions environnantes telles que des résidences d'une part; et l'expérience française en matière de travaux imposés aux riverains pour sécuriser leurs habitations d'autre part. Aujourd'hui, il apparaît difficile d'envisager le départ des industries, en raison du coût inhérent à un tel déplacement, incluant aussi celui de la dépollution des sols; mais aussi de la perte d'emplois et de richesses qu'il susciterait. Il en est de même pour les résidences, constituant le patrimoine de nombreux riverains. Face à ce constat, des réponses peuvent être mobilisées pour diminuer, autant que faire se peut, la vulnérabilité du bâti existant. Il ne s'agit donc pas seulement de voir comment «bâtir au contact du risque ", mais bien de voir comment " agir » sur le bâti déjà en contact avec le risque. Le propos de cet article se découpe en deux parties. Alors que le premier temps de reviendra sur la complexe évolution de la législation en matière de maîtrise de l'urbanisation en France, le second temps portera une focale sur cette réponse particulière qui vise à prescrire aux riverains des travaux de mise en sécurité des habitations.

\section{Des résidences au contact du risque industriel majeur - Une vulnérabilité héritée}

\subsection{Le risque industriel majeur, rare mais dévastateur}

Ulrich Beck, dans son ouvrage La société du risque (2001), affirme que la société, autrefois productrice de richesses, est devenue aujourd'hui productrice de risques. Les risques industriels majeurs sont immatériels et peuvent être vus comme des effets secondaires générés par le développement d'entités pourtant productrices de richesses «palpables» (PERETTI-WATEL, 2000, pp. 75-76). Ces risques constituent un problème collectif et territorial dans la mesure où ils créent une situation qui ne peut être gérée que collectivement et, a fortiori, concernent un large éventail d'acteurs qui cohabitent sur un territoire (RIOUST, 2012, p. 43). En tout état de fait, les industries fabriquant, manipulant ou entreposant des matières dangereuses peuvent connaître des événements tels que des incendies, des fuites toxiques ou bien des explosions. Des événements comme Bhopal (Inde) en 1984, Feyzin (France) en 1966 ou plus récemment Toulouse (France) en 2001, permettent de prendre conscience de la brutalité et des conséquences de tels accidents sur la population, les biens et l'environnement dans son sens le plus large.

\subsection{Des situations de cohabitation entre industries et résidences}

Si par le passé la plupart des industries étaient inscrites dans des espaces peu densément bâtis, il n'en est pas de même aujourd'hui. Certaines industries se sont vues rattrapées par les constructions, en particulier des résidences. En France, il est possible de constater de nombreuses situations où se côtoient ces deux entités. L'image satellite ci-dessous montre des cuves de stockage d'hydrocarbures situées à l'intérieur de l'enceinte de l'aéroport d'Orly en région parisienne. Des résidences, inscrites dans le territoire de la ville riveraine d'Athis-Mons, se situent à proximité directe de l'entité et se révèlent vulnérables à une explosion ou bien à la propagation d'un incendie.

1. Pour Haroun Tazieff, il s'agit de «la menace sur l'homme et son environnement direct, sur ses installations, la menace dont la gravité est telle que la société se trouve absolument dépassée par l'immensité du désastre » (cité dans LARROUY-CASTERA, OURLIAC, 2004, p. 13).

(c) 2016 ISTE OpenScience - Published by ISTE Ltd. London, UK - openscience.fr 


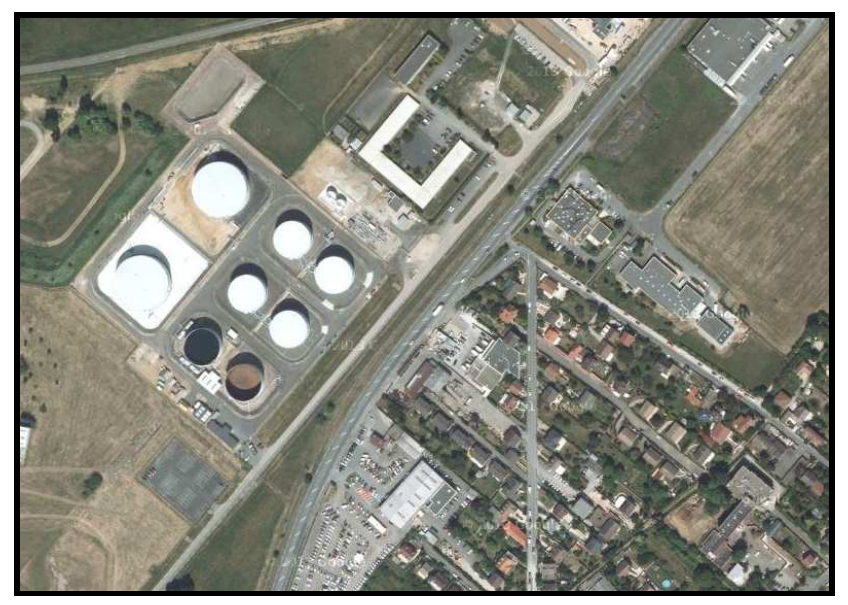

Figure 1.1. Une situation de cohabitation étroite entre industrie et résidences en région parisienne Source : Google-maps.

Pour faire face aux risques inhérents à ce type d'installation et afin de diminuer la vulnérabilité du bâti existant, un arsenal de réponses, notamment règlementaires, est mis en œuvre. Parmi elles, un document planifiant les usages du sol attire l'attention.

\subsection{Le Plan de Prévention des Risques Technologiques (PPRT), instrument d'une politique publique}

Le risque fait l'objet de politiques publiques ; c'est-à-dire d'actions menées par l'autorité publique pour traiter une situation perçue comme un problème (LASCOUMES, LE GALES, 2010a, p. 5 ; HASSENTEUFEL, 2011, p. 8 et 43). En France, il existe une politique publique particulière aux risques industriels majeurs, celle du Plan de Prévention des Risques Technologiques (PPRT) ${ }^{2}$ qui se traduit, entre autres, par un plan de zonage règlementaire. Ce dernier a la particularité d'imposer des mesures foncières et des prescriptions pour certaines constructions à proximité des établissements industriels à la source du risque. Observons un tel plan pour la situation de cohabitation présentée plus haut : 


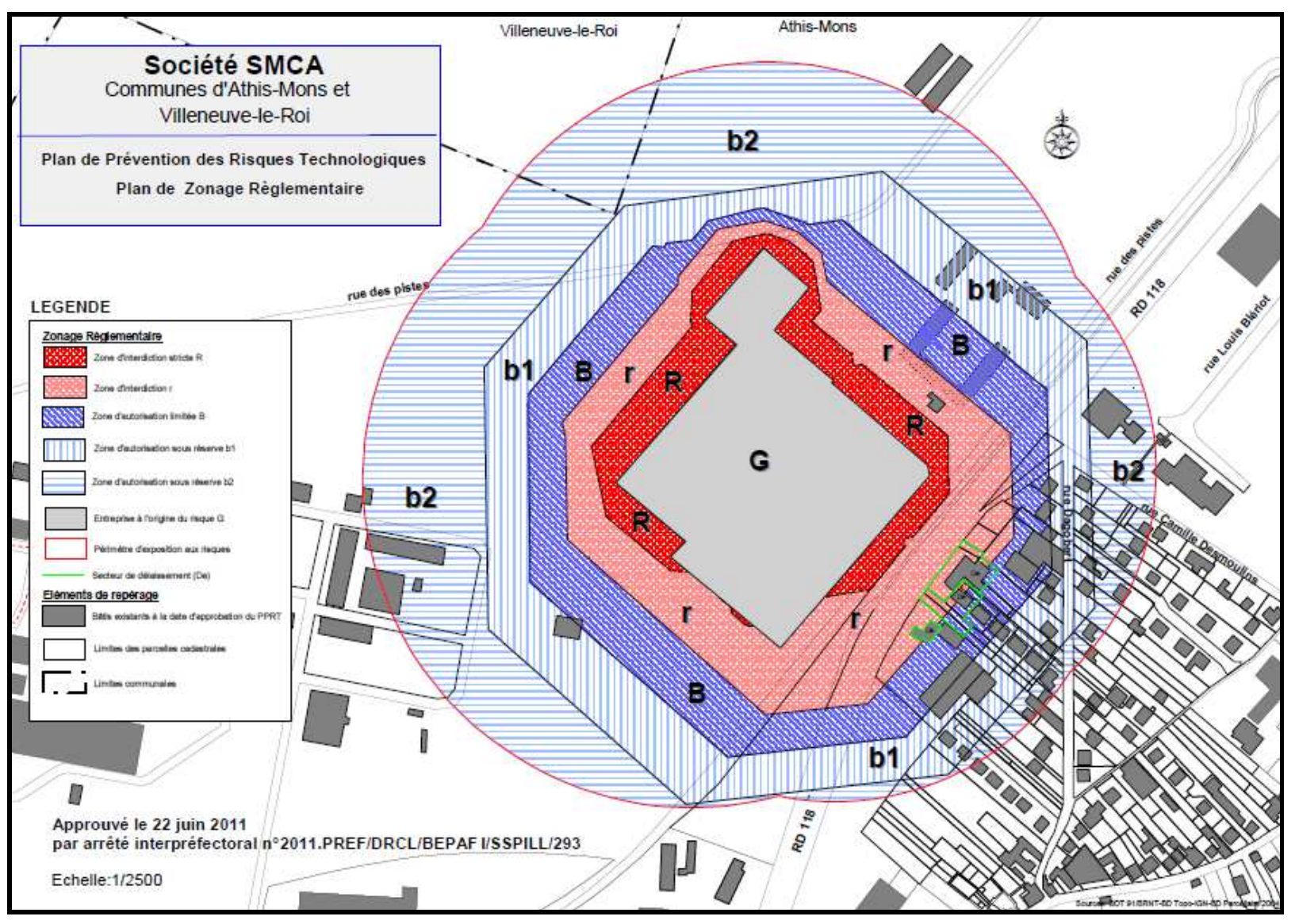

Figure 1.2. Le PPRT - Des conséquences sur les usages du sol Source : Direction Départementale des Territoires (DDT) de l'Essonne (91)

Plusieurs zones peuvent être identifiées. Les zones rouges correspondent aux situations les plus dangereuses. Outre une interdiction de construire, les constructions existantes seront soumises à des mesures foncières qui auront pour objectif de recréer des vides à proximité de l'entité industrielle. Les zones bleues correspondent quant à elles à des secteurs d'autorisations limitées. Si la zone bleue claire est concernée par des recommandations, la zone bleue foncée en revanche est soumise à des prescriptions de travaux pour toutes les constructions. Sans remettre en cause les études techniques qui ont permis la spatialisation de ces zones, il est à remarquer que la frontière entre zones rouges et bleues est assez mince et rectiligne, allant même jusqu'à couper de part en part des constructions. Dans une telle situation, c'est la zone ayant le plus de contraintes qui prend le dessus.

Ce constat est le résultat d'une longue évolution qui débuté au XIX ${ }^{\mathrm{e}}$ siècle. En effet, différents temps dans l'histoire législative française ont structuré la réponse de maîtrise de l'urbanisation

\subsection{Une histoire complexe de la législation française en matière d'aménagement autour des industries à risque}

Il semble que l'existence de catastrophes dans l'histoire d'un pays, en particulier la France, contribue à expliquer les différents temps de sa construction législative (HERAUT, 2004, p. 74). La France est en effet caractérisée par l'existence d'accidents qui ont contribué depuis le début du XIX ${ }^{\mathrm{e}}$ siècle, tels des « chocs exogènes » (HASSENTEUFEL, 2011, p. 263), à façonner l'édifice règlementaire menant à la situation actuelle des PPRT. 


\subsubsection{Au début $d u X I X^{e}$ siècle, la volonté de ne pas entraver le développement industriel}

C'est le décret impérial du 15 octobre 1810 qui marque le début de cette histoire. Ce texte va créer les notions d'établissements classés et d'autorisation préalable en fonction du degré de nocivité de ces derniers. Ce décret, dont le maître mot est l'éloignement, a permis de "procurer un cadre sécurisant à des activités économiques nécessaires au développement national » (SAUVAGE, 1997, p. 19). Il s'agissait donc de ne pas entraver l'essor industriel, de garantir son développement, tout en lui procurant les meilleurs effets possibles (DUCHÊNE, MARTINAIS, 2002, p. 132 ; BONNAUD, MARTINAIS, 2005, p. 2). Un siècle plus tard, la loi du 19 décembre 1917 relative aux établissements dangereux, insalubres ou incommodes vient renforcer la protection des industriels face aux réclamations du voisinage (ibidem, p. 7). Cette période reste largement favorable au développement des industries et à la limitation de son voisinage. Toutefois, des situations de cohabitation entre industries et résidences pouvaient exister à l'image des habitations ouvrières nécessaires pour limiter le déplacement domicile/travail des ouvriers en cette période de faible motorisation; ainsi que pour faciliter l'intervention des ingénieurs en cas de problème (BILLET, 2005, p. 109 ; BAUDELLE, 2001, p.10). La loi du 20 avril 1932 vient néanmoins durcir un aspect, en soumettant certaines catégories d'établissement à la délivrance d'une autorisation dans des zones réservées à l'habitation (IAURIF, 2003, p. 38). Malgré tout, l'intense croissance urbaine rendait difficile l'application des différentes dispositions prises depuis le XIX siècle; et n'ont pu empêcher le rattrapage des industries par les résidences (SAUVAGE, 1993, p. 25 ; CHALINE, DUBOISMAURY, 1994, p. 42).

\subsubsection{L’inflexion des années 1970 - Vers une plus grande prise en compte du voisinage}

C'est à partir du milieu des années 1970 que vont être produits des textes qui prennent en compte, dans une plus grande considération, le voisinage des établissements à risque. Cela s'explique par l'émergence des préoccupations environnementales, ainsi que par des accidents tels que celui de l'explosion de la raffinerie de Feyzin dans le sud de Lyon en 1969 - ce dernier accident ayant fait l'objet d'une importante couverture médiatique - ou bien celui de l'usine chimique de la ville de Seveso (Italie) en 1976 (BONNAUD, MARTINAIS, 2005). Comment dès lors trouver un moyen de permettre la continuation du développement industriel tout en protégeant les espaces environnants ? C'est ainsi que vont émerger deux textes de lois fondamentaux : la loi sur les Installations Classées pour la Protection de l'Environnement (ICPE) du 19 juillet 1976 d'une part ; et celle relative à l'organisation de la sécurité civile, à la protection de la forêt contre l'incendie et à la prévention des risques majeurs du 22 juillet $1987^{3}$ d'autre part.

La loi de 1976 - dont la dénomination d'installations classées est plus positive que celle d'établissements dangereux et insalubres - a pour but de catégoriser les différents établissements en les soumettant à des procédures de déclaration ou d'autorisation auprès du préfet de département. Cependant, le souci de ne pas entraver le développement industriel demeurait en toile de fond, comme en témoigne la volonté d'André Jarrot, alors ministre de la Qualité de la vie lorsqu'il présenta le projet de loi ; ce dernier suggérant «que la législation soit mise en auvre avec une certaine souplesse, notamment en matière d'installations anciennes » (SAUVAGE, 1997, p. 21). La loi de 1987 quant à elle constitue une «rupture dans la façon d'envisager la cohabitation entre la ville et l'industrie » (BONNAUD, MARTINAIS, 2005, p. 11). Elle pose des principes de restrictions foncières aux abords des établissements dangereux dans le but d'améliorer la sécurité des populations tout en préservant le développement industriel (Ibidem, p. 12). Néanmoins, les outils proposés se révélaient plus applicables pour les installations nouvelles (MEDDTL, 2007, p. 19) et non efficients pour des territoires déjà urbanisés (DUBOIS-MAURY et al., 2007, p. 22).

3. A noter que c'est également au cours de cette période que prennent place les deux directives européennes Seveso ; la première datant du 24 juin 1982 et la deuxième datant du 9 décembre 1996. 


\subsubsection{La maîtrise de l'urbanisation à l'horizon des années 2000}

Bien que les années 1980 marquent le temps de l'émergence de la maîtrise de l'urbanisation (MARTINAIS, 2011, p. 79), toute réponse liée à l'aménagement nécessite une identification rigoureuse des dangers. C'est ainsi que deux zones étaient matérialisées autour de l'établissement (LARROUYCASTERA, OURLIAC, 2004, p. 61) :

- La zone Z1 d'une part, dans laquelle un accident aurait des conséquences mortelles pour $1 \%$ des personnes présentes. Cette zone pouvait être inscrite dans les documents d'urbanisme en tant que Zone de Protection Rapprochée (ZPR) à l'intérieur de laquelle aucune construction ne pouvait être autorisée à l'exception des installations industrielles "possédant une culture de sécurité proche de l'installation qui crée le risque, et non susceptibles d'aggraver celui-ci ${ }^{4}$;

- La zone Z2 d'autre part, à l'intérieur de laquelle un accident provoquerait des effets irréversibles pour la santé ou des blessures sérieuses pour les personnes présentes. Les documents d'urbanisme pouvaient traduire cette zone en Zone de Protection Eloignée (ZPE) à l'intérieur de laquelle des constructions étaient autorisées à condition de ne pas augmenter la densité d'occupation du sol.

Malgré l'existence d'instruments mobilisables à l'intérieur de ces zones (servitudes, interdiction de construire), ces derniers se révélaient encore difficilement applicables sur des territoires déjà urbanisés. Une autre limite résidait également dans la négociation dont elles pouvaient faire l'objet. Le passage entre $\mathrm{Z} 1$ et $\mathrm{Z} 2$ et a fortiori ZPR et ZPE tenait compte de l'impératif de sécurité envers les populations ; mais aussi de celui du développement des territoires municipaux; et enfin, des impératifs du fonctionnement des installations. Ainsi, à l'inverse des zones Z1 et Z2 qui ont des contours réguliers, les zones de protection inscrites dans les documents d'urbanisme pouvaient avoir des contours plus irréguliers (SAUVAGE, 1997, pp. 70-71). L'enjeu résidait, par endroit, à obtenir un tracé de zones qui ne contraignaient pas la mise en œuvre de projets urbains (DUCHÊNE, 2001).

En résumé, l'appareil législatif de la France a considérablement évolué depuis le XIX ${ }^{\mathrm{e}}$ siècle, au rythme de certains éléments de contexte, mais surtout à la lumière des retours d'expérience d'accidents industriels (Grenelle, Feyzin, La Mède, et plus indirectement Seveso). Du décret impérial de 1810 à la loi du 19 juillet 1976, en passant par la loi du 19 décembre 1917, un basculement des intérêts semble s'être opéré sans toutefois ôter l'intérêt de l'entreprise toujours prégnant (LASCOUMES, 1994). Croissance industrielle et développement résidentiel semblent s'être tournés le dos jusqu'à la fin du XX ${ }^{\mathrm{e}}$ siècle, si bien qu'aucune disposition ne permettait réellement d'envisager une meilleure cohabitation entre industries et résidences. Il faudra attendre le début des années 2000 pour voir s'engager des réflexions pour qu'industries et résidences puissent se côtoyer de manière plus sécuritaire.

\section{Des réponses pour diminuer la vulnérabilité du bâti existant - Vers le « vivre avec » le risque industriel ?}

C'est suite à l'accident de l'usine AZF à Toulouse en 2001 que la procédure du PPRT a vu le jour en 2003. Ce dernier ne concerne que les établissements classés Seveso « seuil haut» au regard de la directive européenne du même nom. Il s'agit en quelque sorte des établissements considérés comme les plus à risque au regard de la quantité de matières dangereuses manipulées, stockées ou fabriquées (BLESIUS, 2014, p. 58). Les prescriptions de travaux sur le bâti existant en constituent une pierre solide et inédite qui a fait l'objet de nombreux débats et qui a été sujet à une rapide évolution.

4. Circulaire DPPR/SEI/AG.SD du 24 juin 1992 relative à la maîtrise de l'urbanisation autour des installations industrielles à haut risque. 


\subsection{Des interventions obligatoires sur le bâti existant}

L'article L. 515-16 du Code de l'environnement permet au PPRT de prescrire des travaux de sécurisation aux propriétaires de biens se trouvant à proximité de l'installation concernée, dans un délai qui sera déterminé par le plan. Ces travaux ne portent que sur des aménagements dont le coût n'excède pas $10 \%$ de la valeur vénale du bien. Le service d'inspection des installations classées du ministère en charge de l'environnement présente de manière détaillée les mesures qui peuvent être mises en place pour faire face aux différentes familles d'accidents inhérents à ce type d'installation. Pour se prémunir des effets thermiques par exemple, il convient d'intervenir en priorité sur la face la plus exposée à l'entité industrielle. Cela peut passer par la mise en place d'un matériel d'isolation particulier, voire par un remplacement de vitres. Sans prétendre à l'exhaustivité, le tableau suivant présente les mesures qui peuvent être mises en place ainsi que leurs coûts ${ }^{5}$ :

\begin{tabular}{|c|c|}
\hline Type de mesures & Coût indicatif pour une maison individuelle \\
\hline Pose d'un film réfléchissant & $90 €$ à $100 €$ par $\mathrm{m}^{2}$ \\
\hline $\begin{array}{c}\text { Remplacement de fenêtres par des fenêtres } \\
\text { isolantes en bois }\end{array}$ & $\begin{array}{c}440 \text { à } 600 € / \text { unité pour } 1 \text { vantail } \\
660 \text { à } 1000 € / \text { unité pour } 2 \text { vantaux }\end{array}$ \\
\hline $\begin{array}{c}\text { Installation d'une porte d'entrée en bois massif } \\
\text { d'épaisseur } 4 \mathrm{~cm}\end{array}$ & $\begin{array}{c}600 \text { à } 1100 € \text { pour une porte en bois massif } \\
\text { d'entrée de gamme }\end{array}$ \\
$\begin{array}{c}1550 \text { à } 1850 € \text { pour une porte de qualité } \\
\text { supérieure }\end{array}$ \\
\hline $\begin{array}{c}\text { Toiture : isolation thermique laine minérale sous } \\
\text { rampants de toiture }\end{array}$ & $\begin{array}{c}50 \text { à } 70 € / \mathrm{m}^{2} \text { pour une isolation sans } \\
\text { doublage }\end{array}$ \\
$\begin{array}{c}90 \text { à } 135 € / \mathrm{m}^{2} \text { pour une isolation avec } \\
\text { doublage en plaques de plâtre }\end{array}$ \\
\hline $\begin{array}{c}45 \text { à } 70 € / \mathrm{m}^{2} \text { pour une isolation collée }(80 \\
\text { mm d'isolant et } 10 \text { mm de plaque de plâtre }) \\
60 \text { à } 80 € / \mathrm{m}^{2} \text { pour une isolation vissée sur } \\
\text { ossature métallique }-80 \text { à } 100 \text { mm de laine } \\
\text { minérale et plaque de plâtre } 13 \text { mm }\end{array}$ \\
\hline $\begin{array}{c}130 \text { à } 145 € / \mathrm{m}^{2} \text { pour une isolation par } \\
\text { Isolation thermique externe du mur exposé } \\
\text { un enduit minéral de finition résistant au feu } \\
240 \text { à } 420 € / \mathrm{m}^{2} \text { pour une isolation par } \\
\text { l'extérieur de } 80 \text { mm de laine de roche et } \\
\text { parement en terre cuite }\end{array}$ \\
\hline
\end{tabular}

Tableau 2.1. Echantillon de mesures pour faire face aux effets thermiques

Source: Centre d'Etudes et d'Expertise sur les risques, l'environnement, la mobilité et l'aménagement (CEREMA) et Institut Nationale de l'Environnement et des Risques (INERIS), 2014.

Pour se prémunir contre l'effet de surpression, la construction doit être en mesure de résister à une déflagration importante. Comme l'a montré l'accident de l'usine AZF à Toulouse, les fenêtres se révèlent particulièrement vulnérables à ce type d'événement. Il est possible de construire le même type de tableau pour présenter les mesures qui pourraient être mises en œuvre pour sécuriser le bâti existant. Ces dernières doivent permettre à la construction de résister à une pression inscrite entre 20 et 50 mbar.

5. Pour en savoir plus : http://www.amaris-villes.org/actualites-agenda/actualites/289-revue-des-guides 


\begin{tabular}{|c|c|}
\hline Type de mesures & Coût indicatif pour une maison individuelle \\
\hline Pose d'un film de sécurité anti-explosion & $150 € / \mathrm{m}^{2}$ \\
\hline Pose d'un panneau en double vitrage & Entre 400 et $600 € / \mathrm{m}^{2}$ \\
\hline $\begin{array}{c}\text { Remplacement de la fenêtre par une fenêtre } \\
\text { en bois avec système de fermeture à crémone } \\
\text { avec sortie de tringle posé en tunnel }\end{array}$ & $\begin{array}{c}\text { A partir de } 150 € \text { auxquels s'ajoute le coût de } \\
\text { la pose ; à partir de } 300 €\end{array}$ \\
\hline $\begin{array}{c}\text { Remplacement de la fenêtre par une fenêtre } \\
\text { avec système de fermeture adapté }\end{array}$ & $\begin{array}{c}\text { A partir de } 1500 € \text { auxquels s'ajoute le coût } \\
\text { de la pose ; à partir de } 300 €\end{array}$ \\
\hline
\end{tabular}

Tableau 1.2. Echantillon de mesures pour faire face aux effets de surpression Source : CEREMA et INERIS, 2014.

Ces mesures ont certes pour objectif la protection des constructions, mais elles doivent surtout protéger un maximum de vies humaines; tel est l'objectif premier du PPRT. Bien que non abordé au sein de cet article, il existe des mesures qui sécurisent la construction face à l'effet toxique (identification d'une pièce de mise à l'abri, étanchéité des pièces, système de ventilation adapté, etc.).

Malgré les avantages certains que représente la mise en œuvre de ce type de mesures, certaines interrogations restent en suspens dont celle liée au financement de ces différentes opérations. L'ensemble du coût des travaux sur le territoire national a été estimé entre 150 et 200 millions d'euros (ICSI, 2011, p. 6 ; LESQUEL, 2012). Pour atteindre cet objectif, les propriétaires d'un bien immobilier peuvent se voir octroyer un crédit d'impôt (MEDDTL, 2007, p. 44). Si son taux a fait l'objet d'une fluctuation au cours des dernières années (LESQUEL, 2010, p. 20), il est aujourd'hui fixé à $40 \%$ du coût total des travaux effectués (diagnostic préalable et travaux de protection). Il est à noter que ces derniers doivent être effectués par un professionnel et donner lieu à une facture. Si ce point de la loi est aujourd'hui bien accueilli par les élus, la part qui reste à assumer par les riverains a, en revanche, fait l'objet d'approches critiques. C'est ainsi qu'une évolution majeure a eu lieu au cours des dernières années sur le sujet; une évolution qui pourrait constituer un résultat significatif sur la thématique du « vivre avec» le risque industriel.

\subsection{Le combat pour une prise en charge commune des travaux prescrits aux riverains d'une zone industrielle}

L'association nationale des collectivités pour la maîtrise des risques technologiques majeurs (AMARIS) a mené un combat en faveur d'une prise en charge plus globale des travaux prescrits auprès des riverains. Cette structure associative constitue une initiative intéressante à étudier dans la mesure où, à la différence de ce qui a été dit en introduction de cet article, ce sont des municipalités qui ont pris l'initiative de se fédérer et de se donner des missions communes quant aux conditions de vie des individus résidant à proximité d'une installation à risque ${ }^{6}$.

Ceci étant, l'actualité de ces dernières années a été très riche sur la question. Les députés ont en effet voté un amendement au projet de loi de finances de 2013 pour aller dans le sens d'une plus grande prise en charge des travaux prescrits auprès des riverains (BARROUX, 18 décembre 2012) : "Les collectivités et les industriels contribueront à hauteur de 25\% chacun. L'Etat, sous la forme d'un crédit d'impôt, se positionne à hauteur de 40\%. Restent 10\%. La Président de l'association, Yves Blein (également Maire de la Ville de Feyzin), insiste sur la nécessité d'apporter plus d'aide aux riverains qui n'ont souvent pas les 
moyens d'effectuer l'ensemble des travaux ; ces derniers pouvant dépasser les 10000 euros concernant le risque d'explosion (ESQUERRE, 3 décembre 2012). Par ailleurs, ces individus peuvent remettre question la mise en œuvre de tels travaux pour faire face à un danger qui ne leur est pas imputable.

L'amendement au projet de loi de finances de 2013 devait contenir les éléments qui imposent aux installations à l'origine du risque ainsi qu'aux collectivités territoriales percevant tout ou partie de la contribution économique territoriale, de participer aux travaux prescrits dans le cadre des PPRT. La part de chacune des deux parties était fixée à $25 \%$ ce qui, en complément du crédit d'impôt de $40 \%$ en 2013 , laissait 10\% des travaux à la charge de l'individu. L'emploi d'un temps du passé est justifié dans le propos ci-dessus puisque le Conseil constitutionnel a censuré cet élément le 29 décembre 2012 (RADISSON, 3 janvier 2013). Le motif invoqué est que « cette disposition est étrangère au domaine des lois de finances et elle a donc été adoptée selon une procédure contraire à la Constitution (...). Anne Lenormand, en citant le Conseil constitutionnel, indique que cet ajout « $n$ 'avait pas sa place dans une loi de finances, notamment parce qu'il ne concerne " ni les ressources, ni les charges, ni la trésorerie, ni les emprunts, ni la dette, ni les garanties ou la comptabilité de l'Etat »(LENORMAND, 3 janvier 2013).

Néanmoins, les revendications demeuraient présentes, et il faudra attendre la loi du 16 juillet 2013 portant Diverses Dispositions d'Adaptation au Droit de l'Union Européenne dans le domaine du développement durable - dite DDADUE - pour que la prise en charge des travaux prescrits aux riverains à hauteur de $90 \%$ soit adoptée ${ }^{7}$. Les riverains devront donc prendre en charge les $10 \%$ du coût des travaux restant (40\% de crédit d'impôt, $25 \%$ de la part de l'industriel et 25\% de la part de la collectivité).

Ainsi, un basculement semble s'opérer au sein des territoires soumis à des risques industriels majeurs. Industries et résidences peuvent être considérées dans un même ensemble au service de la sécurité. S'il appartient aux industries d'identifier et de diminuer les dangers à la source - tout en faisant preuve de transparence en diffusant des informations au public - il appartient aux riverains de sécuriser leurs habitations. Il semble donc se créer, sur un même territoire, un ensemble d'entités qui œuvrent ensemble pour garantir, autant que faire se peut, un territoire moins vulnérable. Pour autant, il semble que des obstacles doivent encore être franchis pour garantir l'efficience d'une telle démarche.

\subsection{Des obstacles à franchir au service du " vivre avec » le risque industriel ?}

Plusieurs freins au fonctionnement du mécanisme semblent se présenter. C'est tout d'abord la non approbation de tous les PPRT. Bien que la loi imposant leur élaboration date de 2003, il reste encore environ $10 \%$ des PPRT à approuver sur l'ensemble du territoire national ${ }^{8}$. Par ailleurs, il apparaît quelques difficultés dans la mise en œuvre des travaux auprès des riverains. Selon l'association AMARIS, il reste difficile de mobiliser des entreprises pour la réalisation des travaux. A cela s'ajoutent la lenteur de la procédure (décalage entre le diagnostic, la réalisation des travaux et le versement des subventions) ainsi que la question des responsabilités en cas de défaillance. Ce dernier aspect apparaît primordial à éclaircir. Qui serait tenu pour responsable en cas d'accident industriel? Les assurances couvriraient-elles les dommages? Il n'existe pas de retour d'expérience à ce sujet.

Dans tous les cas, un accompagnement des riverains paraît nécessaire. En effet, plusieurs étapes complexes doivent être accomplies. Il convient de produire au préalable un diagnostic des travaux à effectuer avant de les mettre en œuvre; ce qui nécessite de faire appel à un praticien qui possède la compétence technique pour répondre au cahier des charges fixé par le PPRT. Pour accompagner le

7. http://www.actu-environnement.com/ae/news/risques-technologiques-PPRT-financement-loi-Ddadue-travaux-droit-delaissementexpropriation-Amaris-19145.php4

$8 \mathrm{http}: / /$ www.developpement-durable.gouv.fr/IMG/pdf/Liste_PPRT_approuves_2016-02.pdf

(c) 2016 ISTE OpenScience - Published by ISTE Ltd. London, UK - openscience.fr 
riverain dans le montage des dossiers de financement, dans l'élaboration des diagnostics ou bien dans la sollicitation des personnes ressources, une initiative locale a vu le jour. Il s'agit de la démarche des «PARI » (Programmes d'Accompagnement sur les Risques Industriels) lancée par le ministère en charge de l'environnement. Ces programmes, en état d'expérimentation sur quelques sites en France, permettront aux riverains de bénéficier :

- de conseils pour le diagnostic des travaux à entreprendre (devis précis) avec notamment une liste d'artisans qui ont bénéficié d'un programme de formation pour traiter de cette thématique particulière ${ }^{9}$;

- d'un accompagnement pour la réalisation des travaux (constitution des dossiers de demande de subventions ; communication auprès des propriétaires ; contrôle des travaux ; etc.) ;

- d'une aide pour la clarification et la formalisation du montage financier (contribution des différentes parties, règles de versement, temporalité des versements, etc. $)^{10}$.

A termes, ce type de démarche pourrait être couplé avec des opérations d'amélioration de l'habitat qui portent leur focale sur des questions énergétiques. C'est le cas de la vingtaine de sites concernés par la vague d'opérations conjointes «plan de prévention des risques technologiques / amélioration de l'habitat ${ }^{11}$. Il s'agit de rendre compatible l'amélioration de la sécurité du logement d'une part, et l'amélioration de sa qualité d'autre part. Cette démarche permettrait de mobiliser les dispositifs opérationnels de l'Agence Nationale de l'Habitat (ANAH) et d'intégrer les prescriptions de travaux inhérentes aux PPRT. Pour ce faire, le maire, avant de saisir la délégation locale de l'agence, doit s'assurer que le PPRT est approuvé et que le financement a été validé par les différentes parties (industriel, collectivité et Etat). Malgré tout, les financements de travaux propres à la mise en sécurisation du logement ne pourront provenir de l'ANAH. En revanche, associer les deux démarches paraît être avantageux à plus d'un titre; dans la mesure où les interventions pourront être au service d'une meilleure isolation, d'une réduction des bruits extérieurs, d'un plus grand confort, etc. En d'autres termes, les résidences à proximité des industries à risques pourraient voir leur qualité et leur sécurité augmenter conjointement.

\section{Conclusion}

Les réponses mises en œuvre pour faire face aux risques industriels majeurs tendent à conforter une position pourtant délicate à affirmer : la pérennisation, sous conditions, des espaces de cohabitation entre l'industrie et le reste de la ville. Les éléments présentés dans cet article tendent à donner aux PPRT un futur optimiste quant à leur efficience. Bien que les dispositifs soient récents, voire encore au stade d'expérimentation, il semble que les riverains puissent bénéficier d'un accompagnement et d'un financement qui rendront plus facile la mise en œuvre des prescriptions du document règlementaire. Il apparaît par conséquent envisageable de "vivre avec» les industries à la condition que l'ensemble des parties prenantes soient prises en considération et intégrées dans un système de réflexion au service d'une diminution globale des dangers et de la vulnérabilité.

\footnotetext{
9. La liste des artisans est la suivante : http://www.installationsclassees.developpementdurable.gouv.fr/IMG/pdf/Liste_des_diagnostiqueurs_-_05-2014.pdf

10. http://www.amaris-villes.org/

11. http://www.anah.fr/les-actualites/les-actualites/article/prevention-des-risques-experimentations-doperations-conjointes-de-lanah-et-dela-dgrp/

(c) 2016 ISTE OpenScience - Published by ISTE Ltd. London, UK - openscience.fr 
Bien que la législation française réponde à la directive européenne Seveso, elle fait exception quant à l'instrument du PPRT. Ce dernier constitue une originalité dans la mesure où sa focale est portée sur le bâti existant. Au sein d'une recherche récente (BLESIUS, 2014), il a été possible de mener une réflexion sur l'existence d'un instrument similaire au Canada. Bien que d'importantes similitudes existent quant à la mise en œuvre de réponses pour faire face aux risques industriels majeurs, aucune mesure n'est prévue pour traiter des situations de cohabitation héritées du passé.

En tout état de fait, les réflexions semblent plutôt s'orienter vers l'assurance d'une cohabitation sécuritaire entre les industries et le reste de la ville. C'est ce que pense Philippe Audubert, historienurbaniste, qui trouve stimulant de «mettre en valeur les entreprises pour en faire des éléments urbains intéressants » (TARASSIOUX, 2013, p. 17). En effet, au cours du forum sur la sécurité industrielle et la ville durable du 21 mars 2013 de Pierre-Bénite, ce praticien affirme qu'il est possible de mobiliser des techniques architecturales pour que l'industrie retrouve sa place dans la ville. Il propose par exemple de réfléchir à la présence de corridors verts sur un territoire industriel ; au positionnement d'un bâtiment par rapport aux surpressions; à la création de voiles de béton, de talus, d'espaces publics partiellement couverts, etc.

Malgré tout, des questions restent en suspens et sont rattachées à la thématique des représentations des riverains, composante essentielle du risque. En effet, ont-ils conscience d'être à proximité d'une installation à risque ? Acceptent-ils l'existence du PPRT qui les rend responsable de l'exécution de travaux pour faire face à un risque inhérent à une installation ? Par-dessus-tout, ont-ils l'intention de mettre en œuvre les travaux prescrits? Les dispositifs mis en place ne peuvent omettre de porter l'attention sur l'information et l'accompagnement des riverains qui auront peut-être besoin d'être rassurés face au cumul de tous ces éléments (présence d'un risque, obligation règlementaire, résidence en travaux, etc.).

Au total, il semble que c'est par une prise en considération du système dans son ensemble qu'il apparaît possible de parler de résilience. Si cette dernière peut être en partie qualifiée par la robustesse d'un système (MSP, 2008, p. 43), l'auto-organisation, la capacité à trouver des solutions pour faire face à une défaillance, la rapidité d'intervention en cas de perturbation constituent aussi des facteurs clés à prendre en considération (Ibidem; DAUPHINE, PROVITOLO, 2007, p. 117). Les territoires concernés par un risque industriel majeur doivent être capables de souder l'ensemble des maillons d'une chaîne au service d'une diminution globale de la vulnérabilité ; maillons qui tendent encore par endroit à se tourner le dos.

\section{Bibliographie}

AMARIS, «Rapport d'activité », Association nationale des communes pour la maîtrise des risques technologiques majeurs, 2011.

AMARIS, « Restitution synthétique des débats, Quelle place pour le risque technologique dans la ville du XXI ${ }^{\mathrm{e}}$ siècle ? », La Rochelle, 2012.

BARROUX R., «La prévention des risques industriels prend du retard », Le Monde, 18 décembre, 2012.

BAUDELLE G., « Doit-on déménager les usines dangereuses à la campagne ? », Inter Régions, n²39, p. 10-13, 2001.

BECK U., La société du risque. Sur la voie d'une autre modernité, Aubier, Paris, 2001.

BILLET P., «La reconquête foncière des zones exposées à un risque technologique », in CAMPROUX-DUFFRENE M.-P. (dir.), Les risques technologiques - La loi du 30 juillet 2003, Presses universitaires de Strasbourg, p. 107-119, 2005.

BLESIUS J.-C., Vivre avec les industries ? De la maîtrise de l'urbanisation à l'éducation aux risques - Cas de Vitry-sur-Seine (France), Thèse de doctorat, Université de Paris-Est, 2014. 
BONNAUD L. et MARTINAIS E., "Des usines à la campagne aux villes industrielles », développement durable et territoires, dossier 4 : La ville et l'enjeu du développement Durable, http://developpementdurable.revues.org/749, 2005.

CHALINE C., DUBOIS-MAURY J., La ville et ses dangers, Masson, Paris, 1994.

DAUPHINE A. et PROVITOLO D, «La résilience: un concept pour la gestion des risques », Annales de Géographie, $\mathrm{n}^{\circ}$ 654, p. 115-125, 2007.

DIEMER A., LABRUNE S., «L'écologie industrielle : quand l'écosystème industriel devient un vecteur du développement durable », Développement durable et territoires, 2007.

DUBOIS-MAURY J. (dir.), BASSIT R., DOSKOCILOVA-DOLEZEL L. et GOBERT J., «Faisabilité politico-juridique, dans la perspective du développement durable, de dispositifs d'indemnisation, de compensation des risques technologiques, pollutions et nuisances », Programme interdisciplinaire Développement urbain durable, CNRS, Rapport final, 2007.

DUCHÊNE F., «Quand le risque se heurte au territoire : la révision du plan d'occupation des sols autour d'une usine chimique », actes du colloque Risques et territoires, Lyon, p. 109-126, 2001.

DUCHÊNE F., MARTINAIS E., "Les collectivités locales à l'épreuve des risques environnementaux », Annuaire des collectivités locales, Tome 22, p. 129-148, 2002.

ESQUERRE M., «Le président d'Amaris, Yves Blein, pour une refonte du droit du risque industriel - Entretien exclusif », Le Courrier des Maires et des élus locaux, 3 décembre 2012.

http://infos.courrierdesMaires.fr/7377/le-president-damaris-yves-blein-pour-une-refonte-du-droit-du-risque-industrielentretien-exclusif

HASSENTEUFEL P., Sociologie politique : l'action publique, Armand Colin, Paris, 2011.

HERAUT A., De la maîtrise de l'urbanisation et des risques industriels : une impossible équation ?, mémoire de DEA sous la direction de DROBENKO (Bernard), Université de Limoges, 2004.

IAURIF, «Les risques majeurs en Île-de-France - Connaître pour mieux maîtriser », Les Cahiers de l'Institut d'Aménagement et d'Urbanisme de la Région d'Île-de-France, $\mathrm{n}^{\circ} 138,2003$.

ICSI, «Les P.P.R.T. : où en sommes-nous ? 10 questions - Point de vue des élus », Les Cahiers de la sécurité industrielle, 2011.

LARROUY-CASTERA X., OURLIAC J.-P., Risques et urbanisme: risques naturels, risques technologiques, prévention, responsabilités, Editions Le Moniteur, Paris, 2004.

LASCOUMES P., L'éco-pouvoir : environnements et politique, La découverte, Paris, 1994.

LASCOUMES P., LE GALES P., Sociologie de l'action publique, Armand Colin, Paris, 2010.

LENORMAND A., 2013, «PPRT : le dispositif de financement des travaux dans les logements censurés par le Conseil constitutionnel », Localtis.info, 3 janvier 2013.

http://www.localtis.info/cs/ContentServer?pagename=Localtis/LOCActu/ArticleActualiteetjid=1250264646756etcid=125026 4644609

LESQUEL E., 2010, «Risques technologiques et naturels - Comment enfin les prévenir ? ", Le courrier des Maires et des élus locaux, $\mathrm{n}^{\circ} 241$, p. 18-25, 2010.

MARTINAIS E., «L'analyse de risques de 1970 à nos jours. L'histoire d'un instrument d'évaluation des risques industriels particulièrement controversé », "Pour mémoire », revue du ministère de l'écologie, du développement durable, des transports et du logement, Actes de la journée d'étude du 10 novembre 2010:1810-2010:200 ans d'inspection des installations classées, Paris, p. 76-86, 2011.

MEDDTL, Le plan de prévention des risques technologiques (PPRT) - Guide méthodologique, Direction de la Prévention des Pollutions et des risques - Direction Générale de l'Urbanisme de l'Habitat et de la Construction, 2007.

MEDDTL, La démarche française de prévention des risques majeurs, Ministère de l'Ecologie, du Développement durable, des Transports et du Logement, 2011. 
MSP, Concept de base en sécurité civile, Ministère de la Sécurité publique du Québec, Direction générale de la sécurité civile et de la sécurité incendie, Direction du développement, 2008.

NOVEMBER V., Les territoires du risque : le risque comme objet de réflexion géographique, Peter Lang, Berne, 2002.

PERETTI-WATEL P., Sociologie du risque, Armand Colin, Paris, 2000.

RADISSON L. «PPRT: le Conseil constitutionnel censure le dispositif d'aide aux riverains », Actu-Environnement, 3 janvier 2013.

http://www.actu-environnement.com/ae/news/risques-technologiques-PPRT-aides-riverains-PLF-2013-censure-Conseilconstitutionnel-17425.php4

RIOUST E., Gouverner l'incertain : adaptation, résilience et évolutions dans la gestion du risque d'inondation urbaine - Les services d'assainissement de la Seine-Saint-Denis et du Val-de-Marne face au changement climatique, sous la direction de HUBERT G. et DEROUBAIX J.-F., thèse de doctorat, Université de Paris-Est, 2012.

SAUVAGE L., "L’environnement immobilier des industries à haut risque », Etudes foncières, n 61, p. 24-31, 1993.

SAUVAGE L., L'impact du risque industriel sur l'immobilier, ADEF, 1997.

TARASSIOUX S., " Aménager la ville avec les industries - Vivre ensemble, habiter, travailler », Forum sécurité industrielle et ville durable - Entretiens Serge Tarassioux, Pierre-Bénite, 21 mars 2013. 